\title{
Caracterização morfométrica da corvina Micropogonias furnieri (Desmarest) (Pisces, Sciaenidae) na Baía de Sepetiba, Rio de Janeiro, Brasil
}

\author{
Rafaela Nascimento Vicentini ${ }^{1,2}$ \\ Francisco Gerson Araújo 1, 3
}

\begin{abstract}
Morphometric characterization of the white croaker Micropogonias furnieri (Desmarest) (Pisces, Sciaenidae) in Sepetiba Bay, Rio de Janeiro, Brazil. The white croaker Micropogonias furnieri (Desmarest, 1823) is one of the most abundant fish species in the Sepetiba Bay, being heavily exploited in fisheries. Two hundred eleven fishes, captured from October 1998 to September 1999, in monthly samplings with otter trawling, were studied. The aim here is to characterize morphometrics of populations that use de Bay in order to compare with other areas. Measurements included standard length (SL), head length (HL), Pre-dorsal distance (PDD), pectoral distance (PD), ventral distance (VD) and anal distance (AD), pectoral length (PL) and eye diameter (ED). The values of HL, PDD, PD, VD, AD, were related to SL, while ED was related to HL. Scatterplot from logaritmized data, by sex, were analyzed in order to assess alometry. Males outnumber females with differences being highly significant $(\mathrm{p}<0.01)$, mainly in size class 100 and $150 \mathrm{~mm} \mathrm{SL}$. The studied population varying from 62 to $205 \mathrm{~mm}$. Head length varied from 28.7 to $38.6 \% \mathrm{SL}$; PDD 32.5 to $42.9 \%$; PD 27.6 to $41.1 \%$; VD 26.6 to $44.8 \%$; AD 58.1 to $76.8 \%$; and PL 10.8 to $29.8 \%$. Eye diameter varied from 16.7 to $26.1 \% \mathrm{HL}$. Positive alometry $(\mathrm{k}>1)$ were shown for PL in both sexes, and for $\mathrm{AD}$ in female, indicating that these measurements increase proportionately more than the standard length; isometric growth $(k=1)$ were shown for HL, PDD, PD, VD in both sexes, for AD in males; negative alometry $(k<1)$ was shown only for ED in relation to head length (HL), in both males and females. The white croaker populations in the Sepetiba Bay seems to be similar in their morphometrics aspects to the populations of coastal zone at Southeast Brazil, which differ from South coastal zone populations.
\end{abstract}

KEY WORDS. Morphometric, Sciaenidae, coastal fishes, bays, Sepetiba Bay

Micropogonias furnieri (Desmarest, 1823) popularmente conhecida como corvina, é uma das espécies de peixe mais abundante na Baía de Sepetiba, sendo objeto de intensa atividade pesqueira. Apresenta ampla distribuição geográfica, ocorrendo das Antilhas e América Central (Costa Rica) até a Argentina (MENEzES \& FIGUEIREDO 1980). Essa espécie demersal costeira desova em locais próximos a baías e estuários para onde os indivíduos jovens são recrutados e encontram maior disponibilidade de alimento e maior proteção durante o desenvolvimento inicial da vida (VAZZOLER 1991).

1) Laboratório de Ecologia de Peixes, Posto de Aquicultura, Universidade Federal Rural do Rio de Janeiro. Antiga Rodovia Rio-São Paulo, Km 47, 23851-970 Seropédica, Rio de Janeiro, Brasil.

2) Bolsista de Iniciação Científica do CNPq. Email rnvicentini @bol.com.br

3) Pesquisador do CNPq. Email: gerson@ufrrj.br

Revta bras. Zool. 19 (Supl. 1): $163-170,2002$ 
O estudo por métodos numéricos da forma corporal em relação ao tamanho, denominado morfometria, tem se mostrado de grande importância na compreensão da biologia evolutiva dos organismos (BLACKITH \& REYMENT 1971). Os peixes, por apresentarem formas geralmente bem definidas em função do hidrodinamismo, facilitam essa quantificação e se apresentam como objeto de muitos desses estudos (CAVALCANTE \& LOPES 1991).

VAZZOLER (1971), comparando parâmetros populacionais e caracteres morfométricos e merísticos da corvina na plataforma continental sudeste-sul do Brasil, definiu a existência de duas populações: População I: distribuída de $23^{\circ}-29^{\circ}$ S; População II: distribuída de $29^{\circ}-33^{\circ} \mathrm{S}$. Com base nesses dados geográficos a corvina da Baía de Sepetiba se enquadraria na população I, porém são necessários novos estudos para comprovação de tal hipótese, bem como para verificação de eventuais influências de áreas semi-fechadas, como baías e estuários, em relação à zona costeira de plataforma, de onde foram tiradas estas conclusões. ALAMÓN (1983 apud FIGUEROA \& ASTARLOA 1991) estudando exemplares provenientes da área do Chui $\left(33^{\circ}-50^{\circ} \mathrm{S}\right)$ e da Baía de Samborombón $\left(35^{\circ}-50^{\circ} \mathrm{S}\right)$, propôs a existência de diferenças morfológicas entre os indivíduos de ambas regiões. FIGUEROA \& ASTARLOA (1991) observaram um padrão homogêneo entre as localidades do Norte (Chui, Montevideo e Samborombón), que formaram um agrupamento sem limites definidos, diferenciados dos caracteres da população do Sul (Rincón), que constituiu um grupo mais isolado.

Esse trabalho visa apresentar características morfométricas das populações de corvina da Baía de Sepetiba, bem como estabelecer comparações com outras populações de corvina de diferentes latitudes e regiões.

\section{MATERIAL E MÉTODOS}

\section{Área de estudo}

A Baía de Sepetiba é um dos maiores ecossistemas costeiros do Estado do Rio de Janeiro. Apresenta área de $520 \mathrm{Km}^{2}$ e drena uma bacia de aproximadamente 3500 $\mathrm{Km}^{2}$ (Fig. 1). A profundidade na maior parte da Baía varia de $2 \mathrm{a} 12 \mathrm{~m}$, podendo atingir $27 \mathrm{~m}$ no canal de acesso ao Porto de Sepetiba, embora $40 \%$ de sua área seja igual ou inferior a $5 \mathrm{~m}$. Suas águas apresentam temperaturas médias variando entre $20^{\circ} \mathrm{C} \mathrm{e} 29^{\circ} \mathrm{C}$ e salinidade de 22,3 a 34,0 (ARAÚJO et al. 1998). Elevadas pressões de origem antropogênicas ocorrem na área do entorno da Baía devido ao crescente desenvolvimento urbano, associado a um polo industrial que, por carrear seus efluentes para este corpo d'água, contribui aceleradamente para sua degradação (CRUZ-FILHO et al. 2000).

\section{Analise de dados}

Foram analisados 211 exemplares de corvina previamente identificados com base em MENEZES \& FIGUEIREDO (1980), capturados em amostragens mensais com barco do tipo arrasteiro de $12 \mathrm{~m}$ de comprimento, entre outubro de 1998 e setembro de 1999, na Baía de Sepetiba.

Para cada peixe foram tomados os seguintes dados morfométricos com a utilização de um paquímetro (precisão de $0,5 \mathrm{~mm}$ ): comprimento padrão $(\mathrm{Cp})$, comprimento da cabeça $(\mathrm{Cc})$, as distâncias pré-dorsal (Pd), Pré-peitoral $(\mathrm{Pp})$, pré-ventral $(\mathrm{Pv})$ e pré-anal $(\mathrm{Pa})$, o comprimento da nadadeira peitoral $(\mathrm{Cnp})$ e o diâmetro horizontal do olho (Dho) (Fig. 2). 


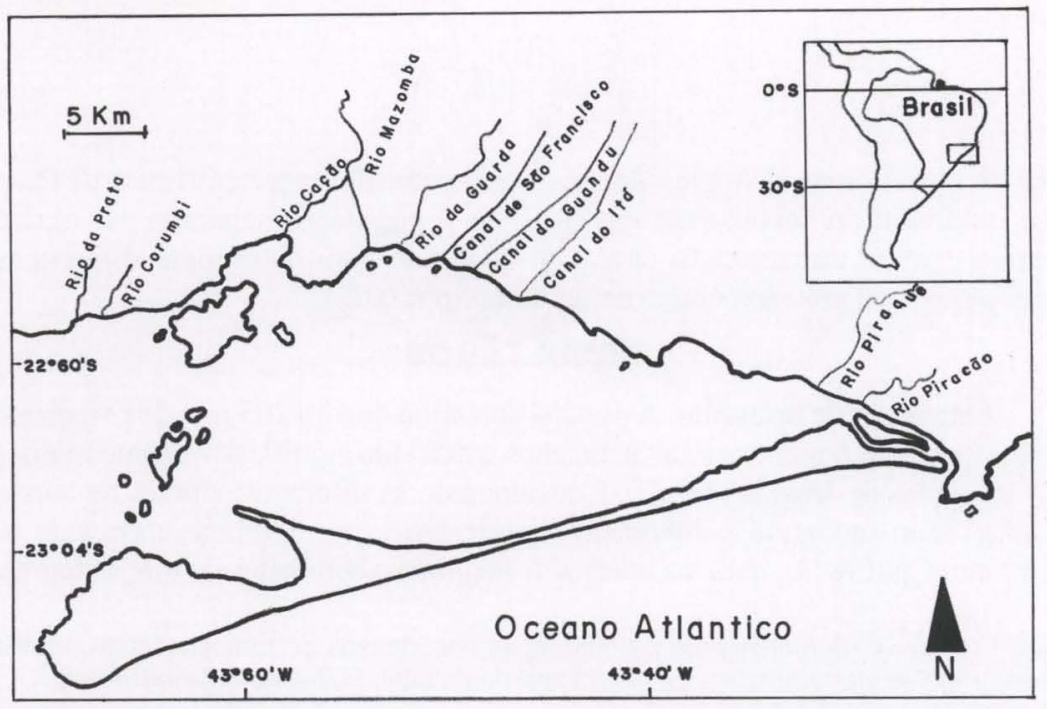

Fig. 1. Mapa da área de estudo. Baía de Sepetiba, Rio de Janeiro.

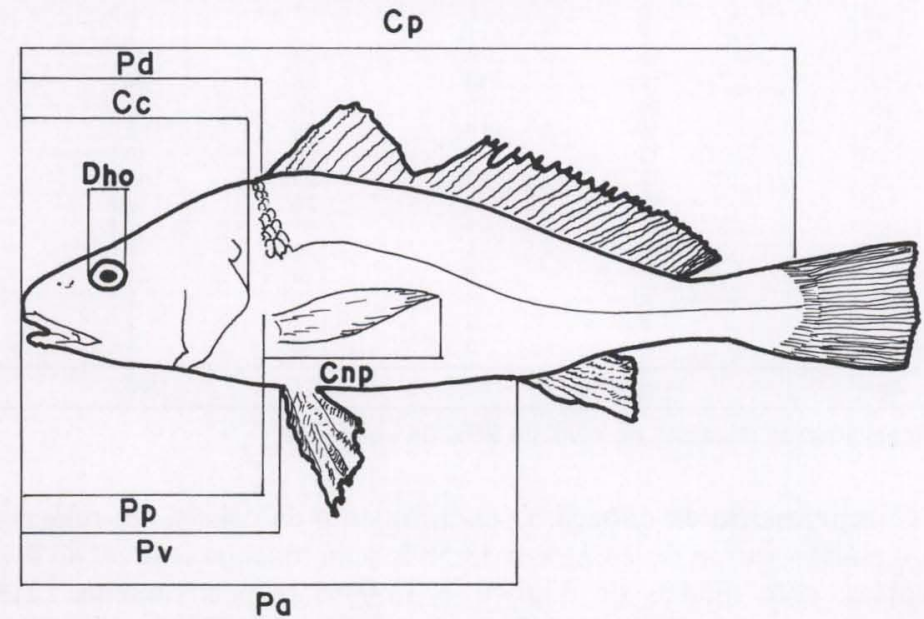

Fig. 2. Caracteres morfométricos tomados de $M$. furnieri: (Cp) comprimento padrão, (Cc) comprimento da cabeça, (Dho) diâmetro horizontal do olho, ( $\mathrm{Pd}$ ) distância pré-dorsal, ( $\mathrm{Pp}$ ) distância pré-peitoral, $(\mathrm{Pv})$ distância pré-ventral, $(\mathrm{Pa})$ distância pré-anal, ( $\mathrm{Cnp}$ ) comprimento da nadadeira peitoral. Desenho esquemático adaptado de MENEZES \& FigueIREDO (1980).

Os valores de $\mathrm{Cc}, \mathrm{Pd}, \mathrm{Pp}, \mathrm{Pv}, \mathrm{Pa}$ e $\mathrm{Cnp}$ foram relacionados ao $\mathrm{Cp}$ em proporções corporais, enquanto que o Dho foi expresso em percentagem do Cc para os sexos em separado. Com os dados logaritimizados foram feitas regressões lineares para cada sexo, e os coeficientes de regressão foram comparados através do teste t-Student (ZAR 1984) a fim de determinar eventuais diferenças de 1 (isometria), através da seguinte equação: 


$$
t=\left[\frac{(b-1)}{\sqrt{\left(1-r^{2}\right)}}\right] *[\sqrt{(n-2)}]
$$

onde: b é o coeficiente de regressão, r é o coeficiente de correlação linear de Pearson e $\mathbf{n}$ é o número de peixes examinados. Com a população separada por classe de tamanho e sexo, utilizou-se o teste Qui-quadrado para determinar diferenças na proporção sexual em cada classe de tamanho $(\mathrm{p}<0,01)$.

\section{RESULTADOS}

Estrutura de tamanho. A população variou de 62 a $205 \mathrm{~mm}$ de comprimento padrão $(\mathrm{Cp})$, com o número total de machos tendo sido significativamente maior $(\mathrm{p}<$ 0,01 ) do que o de fêmeas (Tab. I). Considerando as diferentes classes de tamanho, somente foram encontradas diferenças significativas $(\mathrm{p}<0,01)$ nas classes de 100 e de $150 \mathrm{~mm} \mathrm{Cp} \mathrm{(Fig.} \mathrm{3),} \mathrm{onde} \mathrm{os} \mathrm{machos} \mathrm{foram} \mathrm{mais} \mathrm{abundantes} \mathrm{do} \mathrm{que} \mathrm{as} \mathrm{fêmeas.}$

Tabela I. Teste $X^{2}$ demonstrando as diferenças nas classes de tamanho entre machos e fêmeas. (Fe) Freqüência esperada, (Lt) comprimento total, (n) Número de indivíduos.

\begin{tabular}{cccccc}
\hline $\begin{array}{c}\text { Classes de comprimento Lt } \\
(\mathrm{mm})\end{array}$ & $\begin{array}{c}\text { Machos } \\
(\mathrm{n})\end{array}$ & $\begin{array}{c}\text { Fêmeas } \\
(\mathrm{n})\end{array}$ & $\begin{array}{c}\text { Total } \\
(\mathrm{n})\end{array}$ & Fe & $X^{2}$ \\
\hline 60 & 0 & 1 & 1 & 0,5 & 1,00 \\
70 & 2 & 1 & 3 & 1,3 & 0,33 \\
80 & 14 & 6 & 20 & 10,0 & 3,20 \\
90 & 18 & 10 & 28 & 14,0 & 2,28 \\
100 & 26 & 10 & 36 & 18,0 & $7,11^{*}$ \\
110 & 19 & 9 & 28 & 14,0 & 3,57 \\
120 & 13 & 7 & 20 & 10,0 & 1,80 \\
130 & 17 & 6 & 23 & 11,5 & 5,26 \\
140 & 12 & 3 & 15 & 7,5 & 5,40 \\
150 & 10 & 1 & 11 & 5,5 & 7,36 \\
160 & 8 & 4 & 12 & 6,0 & 1,33 \\
170 & 1 & 2 & 3 & 1,5 & 0,33 \\
180 & 2 & 3 & 5 & 2,5 & 0,20 \\
190 & 2 & 2 & 4 & 2,0 & 0,00 \\
\hline Total & 2 & 0 & 2 & 1,0 & 2,00 \\
\hline
\end{tabular}

*) Significativamente diferente ao nível de $99 \%$ de confiança.

Comprimento da cabeça. O comprimento da cabeça em relação ao comprimento padrão variou de $28,71 \%$ a $38,58 \%$ para machos e de $30,47 \%$ a $37,79 \%$ para fêmeas, com médias de $33,69 \%$ e $33,04 \%$ respectivamente (Tab. II). O coeficiente de regressão (b) foi de 1,019 para machos e 1,067 para fêmeas, não tendo apresentado diferença significativa do valor 1, através do teste t-Student, o que corresponde, portanto, ao padrão de crescimento isométrico do Cc para ambos os sexos (Tab. III; Fig. 4).

Distância pré-dorsal. A distância pré-dorsal variou como percentagem do comprimento padrão de $32,53 \%$ a $42,90 \%$ para machos e de $33,08 \%$ a $41,92 \%$ para fêmeas, apresentando médias de $37,70 \%$ e $36,88 \%$ respectivamente (Tab. II). O coeficiente de regressão (b), com valores de 1,00 para machos e 1,05 para fêmeas, caracterizou crescimento isométrico $(b=1)$ entre essas medidas nos dois sexos (Fig. 5), uma vez que não foram determinadas diferenças significativas através do teste t-Student ( $p>0,01)$ (Tab. III). 


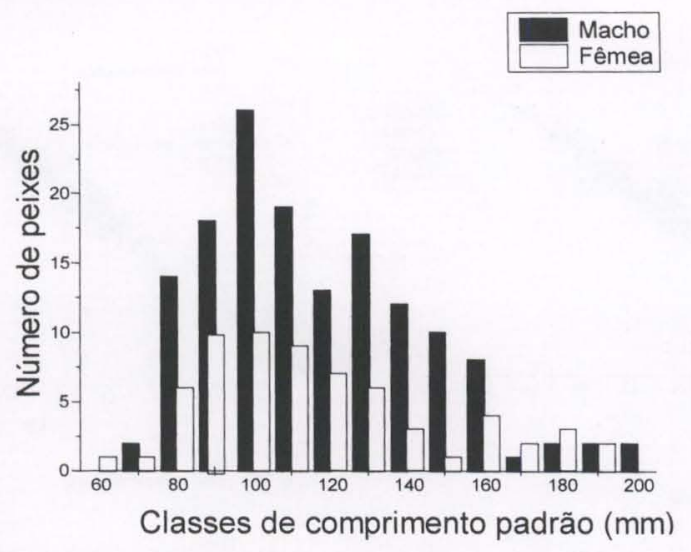

Fig. 3. Freqüência observada por classe de comprimento padrão para os sexos separados de M. furnieri na Baía de Sepetiba, Rio de Janeiro.

Distância pré-peitoral. A distância pré-peitoral apresentou uma variação em relação ao comprimento padrão de $27,70 \%$ a $41,10 \%$ para machos e de $27,65 \%$ a $35,93 \%$ para fêmeas, com médias de $33,04 \%$ e $31,93 \%$ respectivamente (Tab. II). O coeficiente de regressão (b) apresentou valores de 1,01 para machos e 1,07 para fêmeas, não sendo significativamente diferente de 1 ( $\mathrm{p}>0,01)$, caracterizando crescimento isométrico desta distância em ambos os sexos (Tab. III; Fig. 6).

Tabela II. Dados morfométricos de M. furnieri.

\begin{tabular}{|c|c|c|c|c|c|c|c|c|}
\hline \multirow{2}{*}{ Caracteres } & \multicolumn{2}{|c|}{ Menor amplitude } & \multicolumn{2}{|c|}{ Menor amplitude } & \multicolumn{2}{|c|}{ Média } & \multicolumn{2}{|c|}{ Desvio padrão } \\
\hline & Macho & Fêmea & Macho & Fêmea & Macho & Fêmea & Macho & Fêmea \\
\hline C. padrão & 71 & 62 & 205 & 191 & 117,68 & 116,61 & 27,97 & 31,76 \\
\hline \multicolumn{9}{|c|}{ Porcentagens do comprimento padrão } \\
\hline C. cabeça & 28,71 & 30,47 & 38,58 & 37,79 & 33,69 & $33,04^{-}$ & 1,85 & 1,68 \\
\hline D. pré-dorsal & 32,53 & 33,08 & 42,90 & 41,92 & 37,70 & 36,88 & 2,09 & 1,93 \\
\hline D. pré-peitoral & 27,70 & 27,65 & 41,10 & 35,93 & 33,04 & 31,93 & 2,51 & 2,06 \\
\hline D. pré-ventral & 26,59 & 29,72 & 44,88 & 41,40 & 35,90 & 34,75 & 3,52 & 2,86 \\
\hline D. pré-anal & 58,15 & 59,01 & 76,85 & 75,00 & 67,53 & 65,12 & 3,99 & 3,82 \\
\hline C. nad. peitoral & 10,87 & 15,38 & 29,80 & 29,04 & 23,63 & 23,13 & 2,60 & 2,65 \\
\hline \multicolumn{9}{|c|}{ Porcentagens do comprimento da cabeça } \\
\hline D. h. olho & 16,76 & 16,72 & 26,00 & 26,15 & 21,30 & 21,37 & 1,95 & 2,16 \\
\hline
\end{tabular}

Distância pré-ventral. A distância pré-ventral variou de 26,59\% a 44,88\% do comprimento padrão para machos e de $29,72 \%$ a $41,40 \%$ para fêmeas, com médias de $35,90 \%$ e $34,75 \%$ respectivamente (Tab. II). O coeficiente de regressão (b) apresentou um crescimento isométrico não tendo sido encontradas diferenças significativas de 1 para ambos os sexos. (Tab. III; Fig. 7).

Distância pré-anal. A distância pré-anal teve como variação do comprimento padrão um percentual de $58,15 \%$ a $76,85 \%$ para machos, de $59,01 \%$ a $75 \%$ para fêmeas e médias de $67,53 \%$ e $65,12 \%$ respectivamente (Tab. II). O coeficiente de regressão (b) foi diferente entre os sexos, com crescimento isométrico para machos 

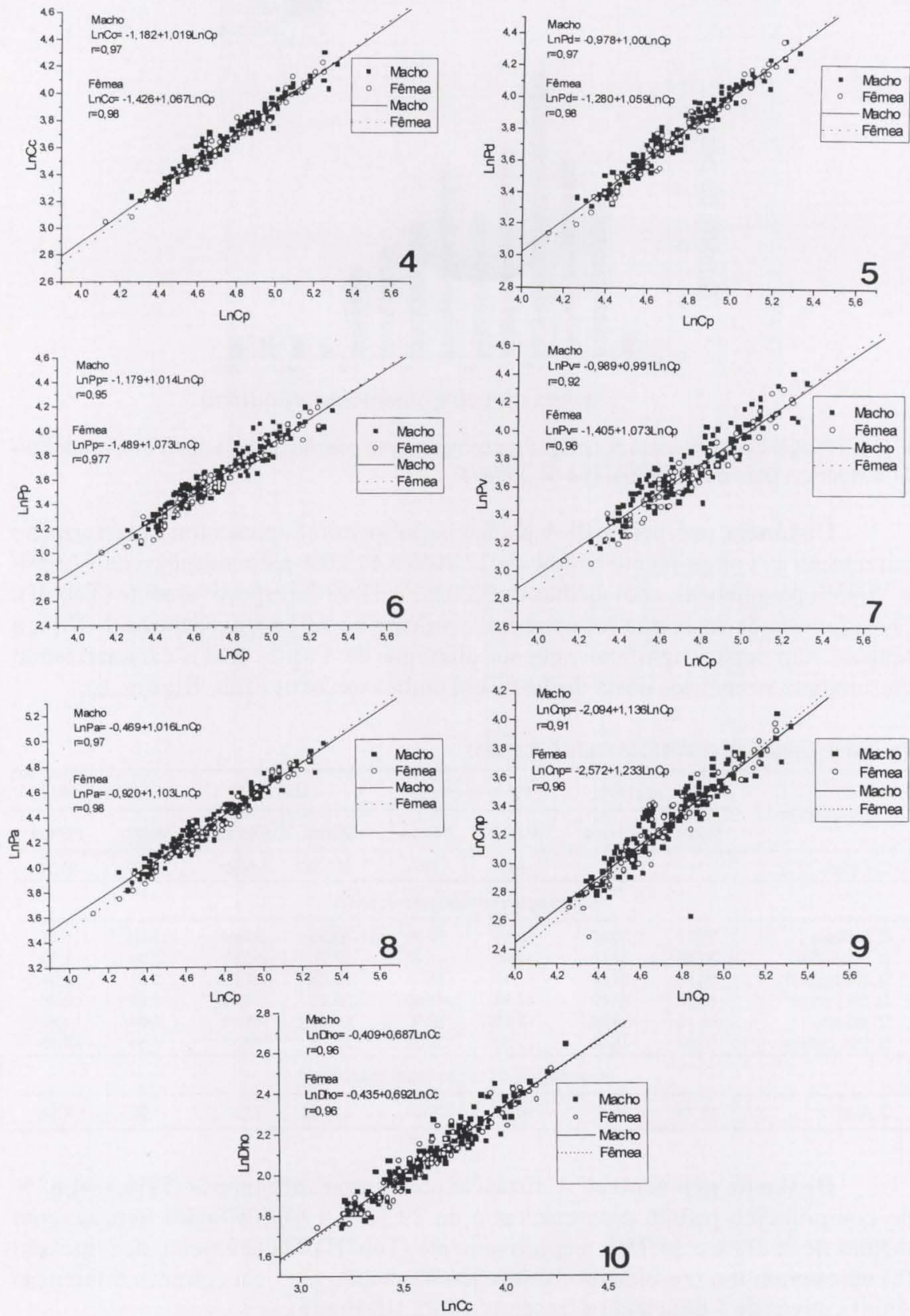

Figs 4-10. Micropogonias furnieri, Baía de Sepetiba, Rio de Janeiro, regressão linear entre os valores logaritmizados do comprimento padrão e: (4) comprimento da cabeça; (5) distância pré-dorsal; (6) distância pré-peitoral; (7) distância pré-ventral; (8) distância pré-anal; (9) comprimento da nadadeira peitoral; (10) diâmetro horizontal do olho. 
$(0,99)$, uma vez que não foi determinada diferença significativa de 1 ( $p>0,01)$, e com alometria positiva para fêmeas $(1,10)$ uma vez que o coeficiente de regressão foi significantemente maior que 1 ( $\mathrm{p}<0,01$ ) (Tab. III; Fig. 8).

Comprimento da nadadeira peitoral. Este caracter apresentou variações de $10,87 \%$ a $29,80 \%$ para machos e de $15,38 \%$ a $29,04 \%$ para fêmeas, em relação ao comprimento padrão. As médias foram de $23,63 \%$ e $23,13 \%$ respectivamente (Tab. II). O coeficiente de regressão, com valores de 1,13 para machos e 1,23 para fêmeas caracterizou alometria positiva para ambos os sexos, uma vez que foram significantemente superiores a 1 ( $\mathrm{p}<0,01)$ (Tab. III; Fig. 9).

Diâmetro horizontal do olho. Em relação ao comprimento da cabeça, esse caracter variou de $16,76 \%$ a $26 \%$ para machos e de $16,72 \%$ a $26,15 \%$ para fêmeas. Suas médias foram de $21,30 \%$ e $21,37 \%$ respectivamente (Tab. II). Foi detectada alometria negativa em ambos os sexos, sendo os valores do coeficiente de regressão 0,68 para machos e 0,69 para fêmeas significantemente menores que 1 ( $\mathrm{p}<0,01$ ) (Tab. III; Fig. 10).

Tabela III. Resultados do teste $t$ aplicado ao coeficiente de regressão para testar diferenças de uma (isometria) das relações entre as medidas ao nível de significância de $p<0,01$. (n) Número de indivíduos.

\begin{tabular}{ccc}
\hline Caracteres & Macho $(n=146)$ & Fềmea $(n=65)$ \\
\hline Cc & 0,494 & 2,383 \\
Dpd & 0,000 & 1,985 \\
Dpp & 0,494 & 1,975 \\
Dpv & $-0,306$ & 1,975 \\
Dpa & 0,494 & $3,971^{*}$ \\
Cpp & $3,762^{*}$ & $6,489^{*}$ \\
Dho & $-13,714^{*}$ & $-8,746^{*}$ \\
\hline Valor Crítico (tc) & 2,62 & 2,66 \\
\hline
\end{tabular}

$\left(^{\star}\right)$ Significativamente diferente ao nível de $99 \%$ de confiança.

\section{DISCUSSÃO}

Crescimento isométrico foi observado para a maioria dos caracteres examinados, com exceção do diâmetro do olho, que diminui proporcionalmente ao comprimento da cabeça, à medida que o peixe cresce, e do comprimento da nadadeira peitoral, que aumenta a sua proporção em relação ao comprimento padrão, o que indica mudanças nestes dois caracteres ao longo do processo ontogenético. Dimorfismo sexual ao longo do desenvolvimento foi observado apenas em relação à distância pré-anal, que para fêmeas aumenta proporcionalmente mais do que o comprimento padrão, enquanto que para os machos essas medidas aumentam na mesma proporção.

Comparada com as populações de M. furnieri do Chui, Montevideo, Samborombóm e Rincon, caracterizadas por FIGUEROA \& ASTARLOA (1991), não foram observadas diferenças nas relações morfométricas na maioria dos caracteres, com exceção do diâmetro do olho e do comprimento da nadadeira peitoral. O coeficiente de regressão apresentou que o diâmetro do olho daquelas populações foi superior 
ao da Baía de Sepetiba, indicando o seu crescimento relativo mais rápido em relação ao comprimento da cabeça, embora ambos tenham apresentado alometria negativa. Com relação ao comprimento da nadadeira peitoral, as populações da Baia de Sepetiba apresentaram maiores coeficientes de regressão, indicando um crescimento mais rápido do comprimento da nadadeira peitoral à medida que os peixes crescem. As populações do Uruguai e da Baía de Sepetiba constituem grupos isolados entre si devido a grande distância entre as duas áreas, portanto, tais diferenças encontradas poderiam estar associadas a esse isolamento.

Os peixes da Baía de Sepetiba apresentaram valores das proporções corporais muito próximos aos da população I definida por VAZZOLER (1971), corroborando a hipótese destes peixes pertencerem à mesma população. VAZZOLER (1971) sugere que em temperaturas mais frias (população da área II) caracteres como comprimento da cabeça e diâmetro do olho são proporcionalmente menores do que em temperaturas mais quentes (população I). Tais diferenças têm sido atribuídas a existência da convergência sub-tropical, uma vez que não existem barreiras biogeográficas bem definidas para separação destas populações.

\footnotetext{
AGRADECIMENTOS. Aos colegas e amigos do Laboratório de Ecologia de peixes da UFRRJ. Este trabalho foi realizado com apoio do Conselho Nacional de Desenvolvimento Científico e Tecnológico, $\mathrm{CNPq}$, através de bolsas e auxílio-pesquisa ao projeto Bioecologia de Peixes da Baía de Sepetiba (Proc. CNPq 522317/96-0 e 46.3317/00-9)
}

\section{REFERÊNCIAS BIBLIOGRÁFICAS}

Araújo, F.G.; A.G. Cruz-Filho; M.C.C. Azevedo \& A.C.A. Santos. 1998. Estrutura da comunidade de peixes demersais da Baía de Sepetiba, RJ. Rev. Brasil. Biol. 58 (3): 417-430.

BLACKITH, R.E. \& R.A. REYMENT. 1971. Multivariate morphometrics. London, Academic Press, 410p. CavalCanti, J.M. \& P.R.D. Lopes. 1991. Morfometria comparada de Ctenosciaena gracilicirrhus, Paralonchurus brasiliensis e Micropogonias furnieri (Teleostei, Sciaenidae) pela análise multivariada de redes de treliças. Revta bras. Zool. 7 (4): 627-635.

Cruz-Filho, A.G. DA; F.G. Araújo; M.C.C. De Azevedo \& A.C. DE A. Santos. 2000. Distribuição de populações de peixes demersais na Baía de Sepetiba, RJ. Acta Biológica Leopoldensia, São Leopoldo, 22 (2): 227-238.

FigueroA, D.E. \& J.M.D. AstARLOA. 1991. Análises de los caracteres morfométricos y merísticos de la corvina rubia (Micropogonias furnieri) entre los $33^{\circ} \mathrm{S}$ y $40^{\circ}$ s (Pisces, Sciaenidae). Atlântica, Rio Grande, 13 (1) 75-86.

Menezes, N.A. \& J.L. Figueiredo. 1980. Manual de peixes marinhos do sudeste do Brasil. IV. Teleostei (3). São Paulo, Museu de Zoologia, 96p.

VAZzoLER, A.E.A. DE M. 1971. Diversificação fisiológica e morfológica de Micropogonias furnieri (Desmarest, 1823) ao sul de Cabo Frio, Brasil. Bol. Inst. Oceanogr., São Paulo, 20 (2): 1-71.

1991. Síntese de conhecimentos sobre a biologia da corvina Micropogonias furnieri (Desmarest, 1823), da costa do Brasil. Atlântica, Rio Grande, 13 (1): 55-74.

ZAR, J.H. 1984. Biostatistical analysis. New Jersey, Prentice Hall, 718p.

Recebido em 08.VI.2001; aceito em 17.VI.2002.

Revta bras. Zool. 19 (Supl. 1): 163 - 170, 2002 